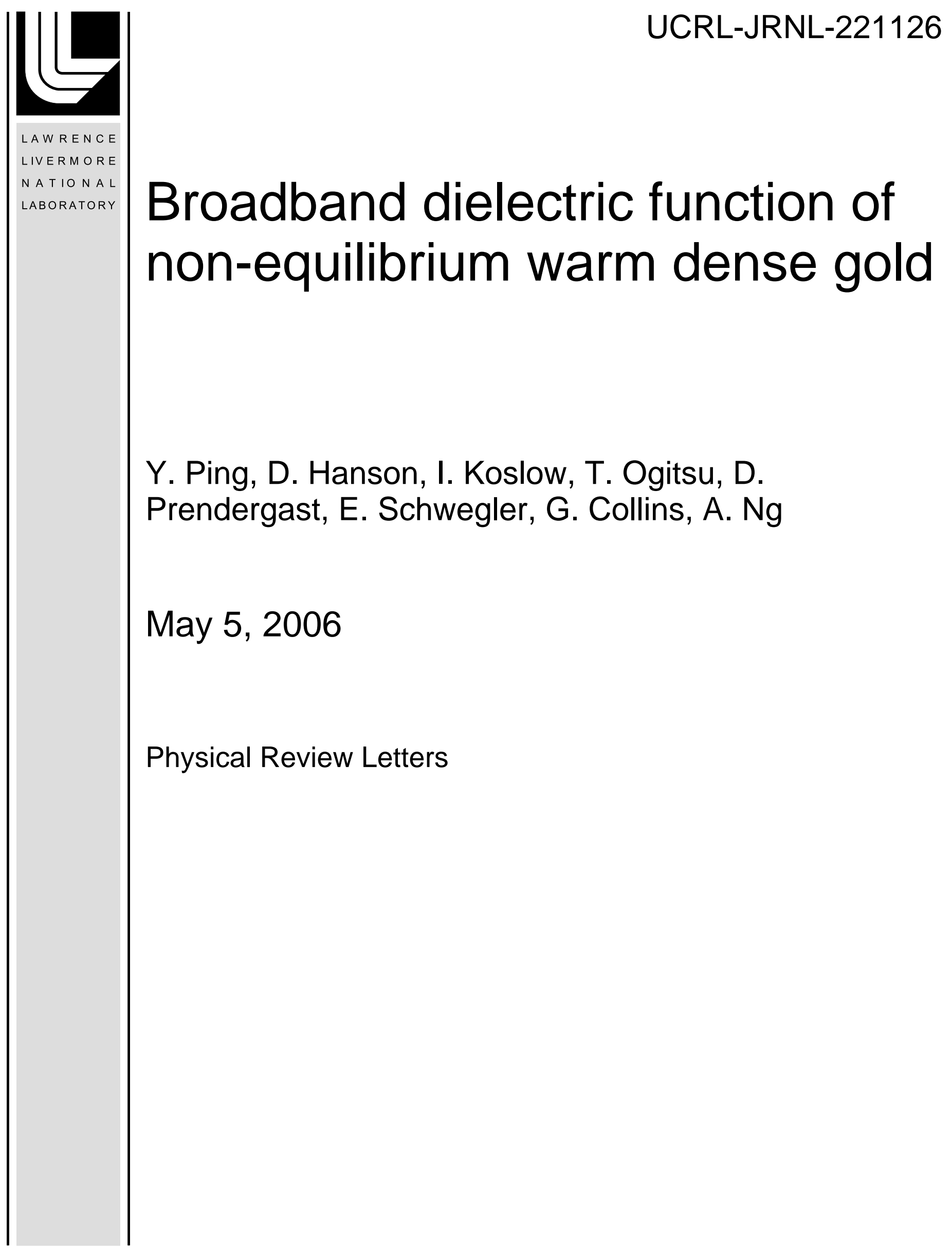


This document was prepared as an account of work sponsored by an agency of the United States Government. Neither the United States Government nor the University of California nor any of their employees, makes any warranty, express or implied, or assumes any legal liability or responsibility for the accuracy, completeness, or usefulness of any information, apparatus, product, or process disclosed, or represents that its use would not infringe privately owned rights. Reference herein to any specific commercial product, process, or service by trade name, trademark, manufacturer, or otherwise, does not necessarily constitute or imply its endorsement, recommendation, or favoring by the United States Government or the University of California. The views and opinions of authors expressed herein do not necessarily state or reflect those of the United States Government or the University of California, and shall not be used for advertising or product endorsement purposes. 


\title{
Broadband dielectric function of non-equilibrium warm dense gold
}

\author{
Y. Ping ${ }^{1}$, D. Hanson ${ }^{2}$, I. Koslow ${ }^{2}$, T. Ogitsu ${ }^{1}$, D. Prendergast ${ }^{1}$, E. Schwegler ${ }^{1}$, G. Collins ${ }^{1}$, and A. Ng${ }^{1}{ }^{2}$ \\ ${ }^{1}$ Lawrence Livermore National Laboratory, Livermore, CA, U.S.A. \\ ${ }^{2}$ Department of Physics \& Astronomy, University of British Columbia, Vancouver, B.C., Canada
}

(Dated: April 27, 2006)

\begin{abstract}
We report on the first single-state measurement of the broadband (450-800 $\mathrm{nm}$ ) dielectric function of gold isochorically heated by a femtosecond laser pulse to energy densities of $10^{6}-10^{7} \mathrm{~J} / \mathrm{kg}$. A Drude and an inter-band component are clearly seen in the imaginary part of the dielectric function. The Drude component increases with energy density while the inter-band component shows both enhancement and red shift. This is in strong disagreement with predictions of a recent calculation of dielectric function based on limited $k$-point sampling.
\end{abstract}

PACS numbers: 52.50.Jm, 52.25.Fi, 52.25.Os, 52.30.-r

Warm dense matter refers to states characterized by comparable thermal and Fermi energies, and ion-ion coupling parameters [1] that exceed unity. While theoretical treatment of warm dense matter is made difficult by effects of electron degeneracy, excited states and strong ion-ion coupling, experimental study is made challenging by the need to obtain single-state data as theory benchmarks. This has left warm dense matter a largely uncharted frontier. Nonetheless, warm dense matter is drawing increasing attention because of its role in understanding the convergence between condensed matter and plasma physics, as well as its relevance to other areas including shock physics [2], inertial confinement fusion [3] and astrophysics [4]. Among this broad interest, a new focus is non-equilibrium warm dense matter. This is driven by its practical importance as transient states in laboratory-produced high energy density matter and its fundamental significance in the study of relaxation processes and phase transitions.

A key parameter characterizing warm dense matter is its dielectric function, $\varepsilon(\omega)$. Encompassing contributions from intra-band and inter-band transitions, it is central to the understanding of electron transport and optical properties. In addition it is a manifestation of band structure and density of state effects, thus rendering it a plausible means of probing structural phases. This is of intense interest to the study of warm dense matter produced not only by laser excitation but also by shock compression. Changes in the imaginary part of the spectral dielectric function in low energy density states have been studied extensively in thermomodulation spectroscopy [5-12] including reflectivity and transmissivity measurements. The only available single-state data on the dielectric properties of highly non-equilibrium warm dense matter are the $\mathrm{DC}$ and $\mathrm{AC}$ conductivities of gold heated by a femtosecond laser to energy densities of $10^{5}$ $10^{7} \mathrm{~J} / \mathrm{kg}$ [13]. However, the latter is measured at a single photon energy of $1.55 \mathrm{eV}$ (wavelength of $800 \mathrm{~nm}$ ) and is thus the result of intra-band transitions. On the other hand, ab initio calculation of the dielectric function of aluminum across the solid-liquid transition has been obtained [14]. Most recently, AC conductivity of warm dense gold in a spectral range that covers both intra- band and inter-band transitions has been reported albeit based on very limited $k$-point sampling [15].

In this letter we present the first measurement of broadband (450-800 $\mathrm{nm}$ ) dielectric function of highly non-equilibrium warm dense gold using a pump-probe technique (femtosecond laser pump and supercontinuum probe [16]). It yields the first single-state data at high energy densities with simultaneous observations of intraband and inter-band components with sub-ps resolution. The results verify Drude-like behavior at $800 \mathrm{~nm}$. They also reveal an enhancement and red-shift of the $d-p$ band transitions that persist until the heated sample disassembles. The measured data provide a critical benchmark for theory and point to the importance of adequate $k$-point sampling to ensure convergence in calculations.

The experiment is performed using two synchronized pulses from a Ti:sapphire laser (the Europa Laser at LLNL). A 150-fs FWHM, 400-nm pulse is used to produce isochoric heating of a free-standing gold nanofoil (25-33 nm thick) while a 180-fs FWHM, 800-nm pulse is focused onto a $\mathrm{CaF}_{2}$ crystal to generate a supercontinuum probe [17] for broadband optical measurements . The gold nanofoil is mounted over an aperture of $600-\mu \mathrm{m}$ diameter. The flatness of its central $300-\mu \mathrm{m}$ diameter is better than $\lambda / 10$ as determined with a $632.8-\mathrm{nm}$ Michelson interferometer. The 400-nm pump pulse is focused onto the nanofoil at normal incidence with a spot diameter of $80 \mu \mathrm{m}$ (FWHM). The incident, reflected and transmitted light are monitored by both calibrated photodiodes and CCD cameras to yield measurements of laser deposition across the focal spot with a spatial resolution of $5 \mu \mathrm{m}$. While the skin-depth of $400-\mathrm{nm}$ light is only $\sim 7 \mathrm{~nm}$ in gold, the electron ballistic range is $\sim 110 \mathrm{~nm}$ [18] that far exceeds the thickness of the target. Combined with the 150 -fs pulse width, this results in uniform and isochoric heating of the nanofoil at solid density and allows the determination of excitation energy density $\Delta \epsilon$ directly from the laser deposition measurement.

The supercontinuum probe illuminates the gold nanofoil at a $45^{\circ}$-incidence with a $30 \mu \mathrm{m} \times 600 \mu \mathrm{m}$ line focus, covering both heated and unheated regions of the target. Figure 1(a) shows a measured supercontinuum spectrum. The frequency chirp in the supercontinuum 

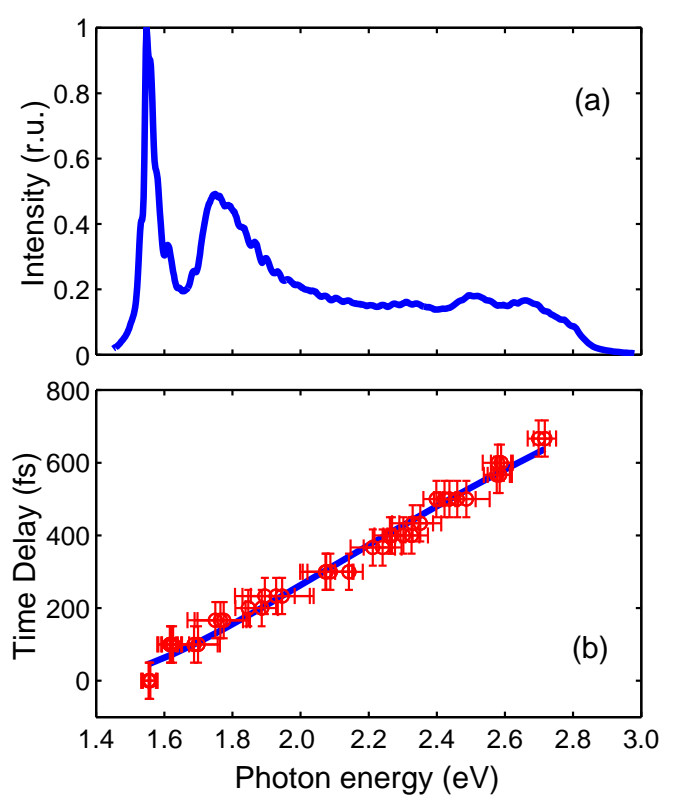

FIG. 1: (a) Supercontinuum spectrum and (b) frequency chip in the supercontinuum probe.

source (Fig. 1(b)) is measured using the Kerr optical-gate technique [19]. To remove the effect of chirp in measurements, spectral data are binned in 10-nm intervals and appropriate temporal shifts are applied using Fig. 1(b). The reflected and transmitted spectra of the supercontinuum probe are recorded with two intensified CCD cameras. Spatially and frequency resolved reflectivity $\mathrm{R}^{*}$ and transmissivity $\mathrm{T}^{*}$ in the heated region are determined using in-situ calibration from the unheated region of the target. These are used to solve the Helmholtz equations for a gradient-free dielectric slab in accordance with the Idealized Slab Plasma concept [20] to yield both the real part, $\varepsilon_{1}(\omega)$, and the imaginary part, $\varepsilon_{2}(\omega)$, of the dielectric function.

Figs. 2(a) and (b) show the temporal evolution of $\varepsilon(\omega)$ of gold at an excitation energy density of $(2.9 \pm 0.3) \times 10^{6}$ $\mathrm{J} / \mathrm{kg}$. The data have been corrected for frequency chirp as described above. Time zero corresponds to the on-set of changes in $\mathrm{R}^{*}$ and $\mathrm{T}^{*}$ from their room temperature values. This is found to be the same for all frequencies in the supercontinuum spectrum after chirp correction. Also included in the figure are tabulated data of gold at room temperature [21]. The minimum energy required for $d-p$ transitions is $\sim 2.3 \mathrm{eV}$. In an earlier study at an excitation energy density of $4.0 \times 10^{6} \mathrm{~J} / \mathrm{kg}$ [22] it was found that the dielectric function at $1.55 \mathrm{eV}$ showed an initial transient consisting of a decrease (increase) in the real (imaginary) part to a minimum (maximum) value in $\sim 600$ fs. This was followed by an increase (decrease) to a quasi-steady-state value in $\sim 900 \mathrm{fs}$. The quasi-steady state then lasted for another $\sim 4$ ps before apparent target disassembly occurred. The time steps in Fig. 2 are chosen to span a similar duration. The new data at 1.55

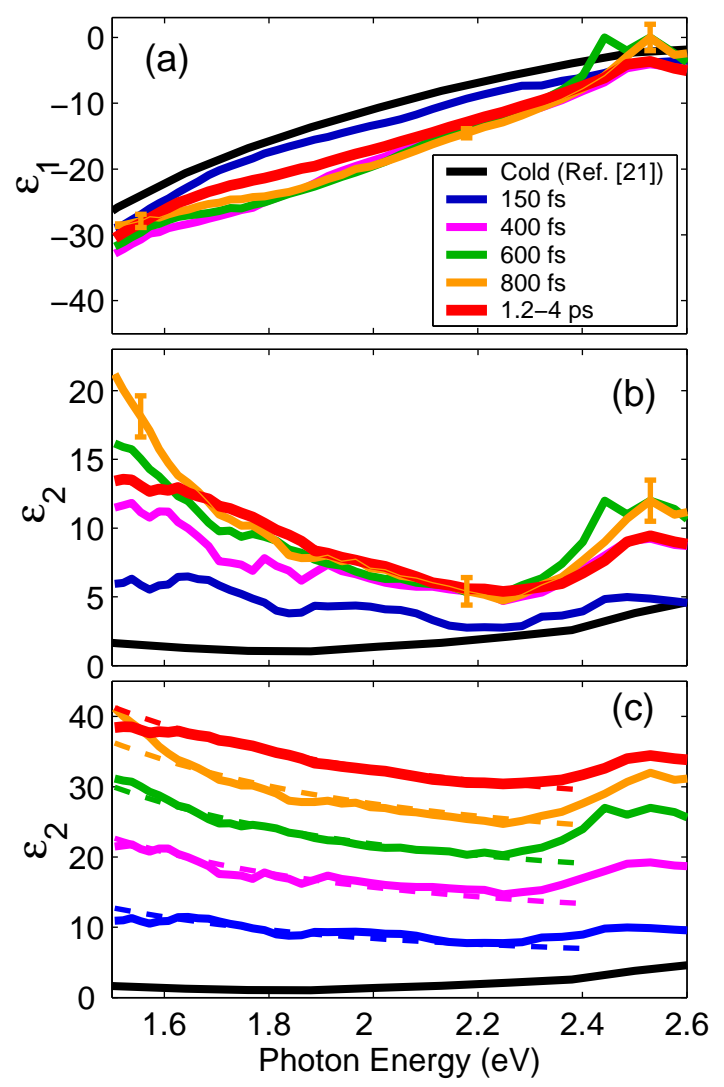

FIG. 2: (a) $\varepsilon_{1}(\omega)$ and (b) $\varepsilon_{2}(\omega)$ at different times, and (c) $\varepsilon_{2}(\omega)$ displayed with an off-set of +5 along the $y$-axis between time steps.

eV show similar transient and quasi-steady-state behaviors as described above. A single $\varepsilon(\omega)$ plot is presented for $1.2-4$ ps since no significant change in $\varepsilon(\omega)$ is observed in the interval, consistent with the quasi-steady state found in a previous study [22].

For the observed spectral range, $\varepsilon_{1}(\omega)$ appears relatively featureless. However, intra-band and inter-band $(d-p)$ components are clearly discernable in $\varepsilon_{2}(\omega)$ below and above $\sim 2.3 \mathrm{eV}$. They also show substantial enhancements over their room-temperature values. Furthermore, by displaying $\varepsilon_{2}(\omega)$ at different time steps with an offset (Fig. 2(c)), it can readily be seen that the intra-band component shows good agreement with best-fitted Drude functions [23] that assume frequency-independent electron collision time and density, except for a small region around $1.6 \mathrm{eV}$ at the peak of the transient at $800 \mathrm{fs}$. The fitting parameters are given in Table 1.

To examine the dependence of $\varepsilon(\omega)$ on the excitation energy density $\Delta \epsilon$, we use measurements made on the quasi-steady state. The results are presented in Fig. 3. These are not corrected for frequency chirp and the probe delay varies from $1.4 \mathrm{ps}$ at $1.55 \mathrm{eV}$ to $2 \mathrm{ps}$ at $2.6 \mathrm{eV}$. Time zero again corresponds to the onset of observed changes in $\mathrm{R}^{*}, \mathrm{~T}^{*}$ at $1.55 \mathrm{eV}$. For $\Delta \epsilon$ of $2.2 \times 10^{6}$ and $4.7 \times 10^{6}$ $\mathrm{J} / \mathrm{kg}$, the 1.4-2 ps probe delay falls completely within the quasi-steady state duration [22] allowing the dielec- 

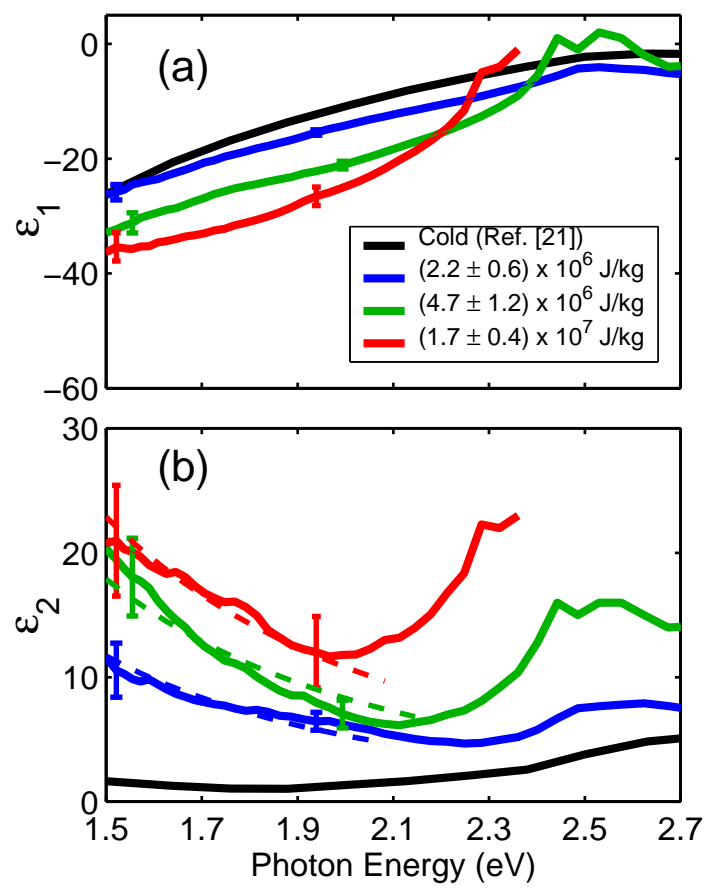

FIG. 3: (a) $\varepsilon_{1}(\omega)$ and (b) $\varepsilon_{2}(\omega)$ for different excitation energy densities.

tric function to be determined over the entire spectral range of $1.55-2.6 \mathrm{eV}$. However, at $1.7 \times 10^{7} \mathrm{~J} / \mathrm{kg}$ no solution to the Helmholtz equations can be found for $\varepsilon(\omega)$ from the $\mathrm{R}^{*}, \mathrm{~T}^{*}$ data above $2.38 \mathrm{eV}$. This is due to the breakdown of the uniform slab assumption as target disassembly gives rise to gradients in the expanding foil. The cut-off point at $2.38 \mathrm{eV}$ corresponds to a probe delay of 1.9 ps that is consistent with the disassembly time observed in the earlier experiment [22]. Accordingly, dielectric function measurement using a frequency chirped source offers a new means of probing hydrodynamic disassembly of a heated solid.

With increasing excitation energy density, the quasisteady state $\varepsilon_{1}(\omega)$ begins to reveal the effect of interband $(d-p)$ transitions above $2.1 \mathrm{eV}$ while $\varepsilon_{2}(\omega)$ exhibits increasing enhancements in intra-band and inter-band transitions. The intra-band component continues to be Drude-like as indicated by the best-fitted Drude functions in the figure. The fitting parameters are also given in Table 1. It should be noted that the quasi-steady state collision time and electron density are in good agreement with previous measurements [13]. The inter-band component also shows a red shift that increases with excitation energy density.

$A b$ initio calculations of the AC conductivity of warm dense states of gold have been reported recently by Mazevet et al. [15]. Their results show a highly structured frequency spectrum with a local maximum at 1.5 $\mathrm{eV}$ and the absence of any well-defined red shift or enhancement of the inter-band component above $2 \mathrm{eV}$. This deviates substantially from our observation described above. To understand the cause for the discrepancy, we
TABLE I: Parameters for Drude fitting in Figs. 2 and 3.

\begin{tabular}{ccccc}
\hline \hline & $\Delta \epsilon\left(10^{6} \mathrm{~J} / \mathrm{kg}\right)$ & Probe Delay & $\tau\left(10^{-15} \mathrm{~s}\right)$ & $\mathrm{n}_{e}\left(10^{22} \mathrm{~cm}^{-3}\right)$ \\
\hline (a) & 2.90 & $150 \mathrm{fs}$ & 1.49 & 4.54 \\
(b) & 2.90 & $400 \mathrm{fs}$ & 1.16 & 6.01 \\
(c) & 2.90 & $600 \mathrm{fs}$ & 0.97 & 6.34 \\
(d) & 2.90 & $800 \mathrm{fs}$ & 0.87 & 6.45 \\
(e) & 2.90 & $1.2-4 \mathrm{ps}$ & 0.83 & 5.93 \\
(f) & 2.20 & $1.4-2 \mathrm{ps}$ & 1.00 & 5.10 \\
(g) & 4.70 & $1.4-2 \mathrm{ps}$ & 0.86 & 7.00 \\
(h) & 17.0 & $1.4-2 \mathrm{ps}$ & 0.77 & 8.40 \\
\hline \hline
\end{tabular}

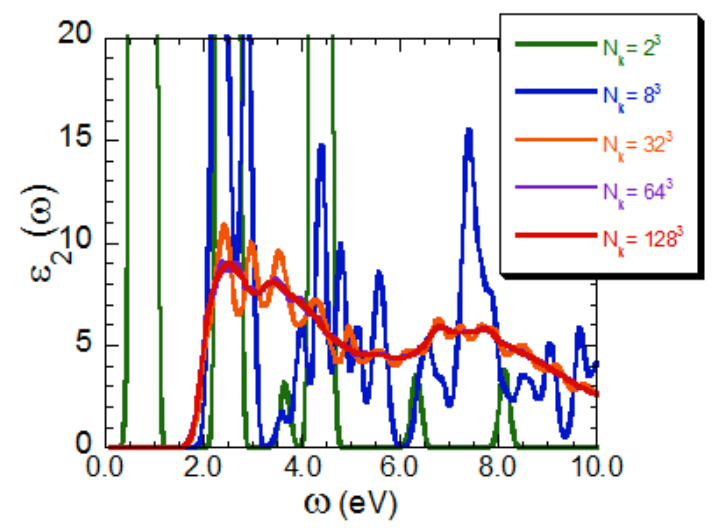

FIG. 4: $\varepsilon_{2}(\omega)$ of gold at $0 \mathrm{~K}$ calculated with different $k$-point sampling.

calculate $\varepsilon_{2}(\omega)$ based on density functional theory within local density approximation [24]. Our focus is on the convergence of the calculation in terms of finite size effect on the electronic degrees of freedom, i.e., on $k$-point sampling. Troullier-Martin's optimized pseudopotential is used to represent the ionic core potential with a plane wave expansion truncated at a cutoff energy of $35 \mathrm{Ry}$. The lattice constant is kept fixed at 7.657 au since earlier measurements indicate the absence of significant expansion during the quasi-steady state of the gold foil [13, 22]. $\varepsilon_{2}(\omega)$ is then calculated using the Kubo-Greenwood formula [25] where the atomic core region of wavefunctions are replaced by atomic orbitals to avoid complication arising from the non-locality of the pseudopotential [26].

A crucial aspect of such an $a b$ initio approach is the choice of the $k$-point mesh that is known to affect the accuracy in calculating inter-band contributions to the dielectric function [14]. This is because the characteristic transition peaks originate from a small area in the Brillouin zone where branches of the bands become parallel to each other. In our calculation of $\varepsilon_{2}(\omega)$ for a perfect fcc gold crystal at $0 \mathrm{~K}$, convergence is reached at a $128^{3}$ uniform $k$-points grid as shown in Figure 4 . The result is in excellent agreement with that obtained from an all-electron Linear-Augmented-Plane-Wave (LAPW) investigation [27]. This indicates that for a 32-atom gold system, sampling over $65,000 k$-points is required to ensure fidelity of the calculation. It is evident from Fig. 
4 that insufficient $k$-point sampling can readily generate substantial fluctuations in the dielectric function. This may account for the highly structured conductivities seen in previous calculations of a 32 -atom system with sampling over only $4 k$-points [15], which roughly corresponds to $8^{3} k$-points with a FCC unit cell. It may also contribute to the spurious local maximum seen at $1.54 \mathrm{eV}$ giving it a non-Drude appearance.

In conclusion, our new measurements have given us some interesting insights to the behavior of ultrafast laser excited gold. A basic property contained in the spectral dielectric function is electron density of state. Thus, the data in Fig. 2 carry information on the evolution of the density of the $d$ and $s / p$ states driven by the processes of photo-excitation of $d$-electrons, electron-hole recombination and electron-electron thermalization at high energy densities. Similarly, the data in Fig. 3 carry information on the dependence of density of state on excitation energy density. For the range of conditions of interest, Drudelike behavior of the intra-band component of $\varepsilon_{2}(\omega)$ is confirmed. This provides a crucial validation for the use of AC conductivity at $800 \mathrm{~nm}$ to derive DC conductivity, collision time and carrier density of warm dense gold [13], thus significantly enhancing the utility of AC conductivity measurement. The appearance of inter-band transitions presents the first evidence of the persistence of $d$-band in the quasi-steady state of ultrafast laser heated gold. If it can be assumed that the presence of a $d$-band can only result from long-range ordering, this raises the possibility that the quasi-steady state is the characteristic of a super-heated solid which would be consistent with a recent empirical two-temperature model [15]. Equally important are the observed increases in red shift and en- hancement of $d-p$ transitions with excitation energy density. Red shifts in the inter-band transition region may result from temperature-induced changes in the FermiDirac distribution of the electrons. The enhancement is however unexpected. Calculations of optical absorption spectrum of equilibrium solid and liquid phases of aluminum [14] show only red shifts in inter-band transitions but no enhancements as the solid is heated to its melting point, consistent with observation [28]. On the other hand, measurements of electron energy distribution function of ultrafast laser excited gold [29] have shown that even at very low excitation energy densities $\left(300 \mu \mathrm{J} / \mathrm{cm}^{2}\right.$ or equivalently $\sim 5 \times 10^{5} \mathrm{~J} / \mathrm{kg}$ for a 30 -nm-thick gold foil), a small energetic electron tail appears to remain visible $670 \mathrm{fs}$ after the laser pump pulse. We are thus led to conjecture that our observed enhancements in $d-p$ transitions are likely the manifestation of non-equilibrium density of state resulting from the complex processes of electronhole recombination and electron-electron relaxation in states driven to very high energy densities.

In addition, our data have provided the first benchmark for testing theory [15]. We believe that insufficient $k$-point sampling is the likely cause for the noted discrepancy between theory and experiment. This points to the need for examining proper convergence of numerical calculations with regard to finite-size effects.

We wish to thank L. Bendict for valuable discussions. This work was performed under the auspices of the U.S. Department of Energy by University of California Lawrence Livermore National Laboratory under Contract No. W-7405-ENG-48 and was supported by the Natural Sciences \& Engineering Research Council of Canada.
[1] S. Ichimaru, Rev. Mod. Phys. 54, 1017 (1982).

[2] A. Ng et al., Laser \& Particle Beams 23, 527 (2005).

[3] T.R. Dittrich et al., Phys. Plasmas 6, 2164 (1999).

[4] D. Saumon et al., High Pressure Res. 16, 331 (2000).

[5] W.J. Scouler, Phy. Rev. Lett. 18, 445 (1967).

[6] R. Rosei and D.W. Lynch, Phys. Rev. B 5, 3883 (1972).

[7] R. Rosei et al., Surface Science 37, 689 (1973).

[8] R. Rosei, Phys. Rev. B 10, 474 (1974).

[9] E. Colavita et al., Phys. Rev. B 20, 4864 (1979).

[10] J.E. Nestrell Jr. et al., Phys. Rev. B 21, 3173 (1980).

[11] E. Colavita et al., Phys. Rev. B 27, 4684 (1983).

[12] H.E. Elsayed-Ali and T. Juhasz, Phys. Rev. B 47, 13599 (1993).

[13] K. Widmann et al., Phys. Rev. Lett. 92, 125002 (2004).

[14] L. X. Benedict, J. E. Klepeis and F. H. Streitz, Phys. Rev. B 71, 064103 (2005).

[15] S. Mazevet et al., Phys. Rev Lett. 95, 085002 (2005).

[16] L. Huang, J. P. Callan, E. N. Glezer and E. Mazur, Phys. Rev. Lett. 80, 185 (1998).

[17] R. Huber, H. Satzger, W. Zinth and J. Wachtveitl, Opt. Commun. 194, 443 (2001).
[18] J. Hohlfeld et al., Chem. Phys. 251, 237 (2000).

[19] C. Nagura et al., Applied Optics 41, 3735 (2002).

[20] A. Forsman et al., Phys. Rev. E 58, R1248 (1998).

[21] P. B. Johnson and R. W. Christy, Phys. Rev. B 6, 4370 (1972).

[22] T. Ao et al., Phys. Rev. Lett. 96, 055001 (2006).

[23] P. Drude, Ann. Phys. (Leipzig) 1, 566 (1900).

[24] D. M. Cepewrley and B. J. Alder, Phys. Rev. Lett. 45, 556 (1980). J. P. Perdew and A. Zunger, Phys. Rev. B 23, 5048 (1981).

[25] R. Kubo, J. Phys. Soc. Japan 12, 570 (1957). D. A. Greenwood, Proc. Phys. Soc. (London) A71, 585 (1958).

[26] H. Kageshima and K. Shiraishi, Phys. Rev. B 56, 14985 (1997).

[27] P. Romaniello and P. L. de Boeij, J. Chem. Phys. 122, 164303 (2005).

[28] A.G. Mathewson and H.P. Meyers, J. Phys. F: Met. Phys. 2, 403 (1972).

[29] W. S. Fann et al., Phys. Rev. B 46, 13592 (1992). 